\title{
Szenarien künftiger nachhaltiger Entwicklung
}

\author{
Andreas Klee $\cdot$ Hendrikje Wehnert
}

Online publiziert: 18. September 2013

(C) Springer-Verlag Berlin Heidelberg 2013

Nachhaltigkeit ist ein Wort, das mittlerweile einem Großteil der Bevölkerung ohne zu zögern über die Lippen geht. Wir reden von Nachhaltigkeit in der Produktion, lesen Zeitschriften zur nachhaltigen Entwicklung, investieren in nachhaltige Projekte, bauen nachhaltig und führen einen nachhaltigen Lebensstil. Man könnte bei oberflächlicher Betrachtung meinen, dass die Nachhaltigkeit auf breiter Front und quasi von allein Einzug in unser modernes Leben gehalten hat.

Doch auch die Bewusstseinsentwicklung für Nachhaltigkeit speist sich unter anderem aus der Politik. Die Steuerungspotenziale der Agrar- und Raumordnungspolitik im Hinblick auf die in ihnen formulierten Zielsetzungen der nachhaltigen Entwicklung ländlicher Regionen betrachten Kaufer/Krott/Hubo/Giessen in ihrem wissenschaftlichen Beitrag. Sie analysieren dazu Gesetze, politische Erklärungen, Strategien und Pläne auf europäischer, nationaler und regionaler Ebene, leiten die implizierten Ziele ab und ermitteln die konkreten politischen Instrumente, die zur Erreichung der Ziele zur Verfügung stehen. Die Autoren stellen fest, dass starke Instrumente mehrheitlich für die Nachhaltigkeitsdimensionen „Ökonomieorientierung“ und „globale Orientierung“ installiert wurden. Die Nachhaltigkeitsdimensionen „Ökologieorientierung“ und „regionale

H. Wehnert $(\bowtie)$

Leibniz-Institut für ökologische Raumentwicklung, Weberplatz 1, 01217 Dresden, Deutschland

E-Mail: h.wehnert@ioer.de

Dr. A. Klee

Akademie für Raumforschung und Landesplanung - LeibnizForum für Raumwissenschaften, Hohenzollernstraße 11, 30161 Hannover, Deutschland

E-Mail: klee@arl-net.de
Orientierung“, für die ebenfalls anspruchsvolle Ziele formuliert werden, müssen jedoch ohne instrumentelle Unterstützung auskommen.

In diesem Heft finden sich drei Berichte aus Forschung und Praxis. Wir freuen uns, dass wir Ihnen in dieser Rubrik zwei Berichte zur Szenarioplanung nebeneinander vorstellen können. Hauck/Priess berichten von der Entwicklung sogenannter Storylines unter dem Titel „The most likely future isn't: Landnutzungsszenarien für Mitteldeutschland“". In Zusammenarbeit mit einem interdisziplinär besetzten Review-Panel, mit unterschiedlichen Praxispartnern in Workshops und begleitenden Expertenrunden wurden Szenarien in Bezug auf zwei mögliche wirtschaftlich-politische Entwicklungsrichtungen (marktwirtschaftliche Orientierung, Nachhaltigkeit und bürgernah) erstellt. Sie wurden unter Einbeziehung wichtiger regionaler Komponenten sowie in zwei Intensitäten (extrem/moderat) entwickelt. Ausführlich werden die Szenarien in Bezug auf die Entwicklung beispielsweise des Verkehrs, der städtischen und ländlichen Räume sowie der Energienutzung im Zeithorizont 2050 verbal und mittels anschaulicher Karten dargestellt.

Ebenfalls auf 2050 beziehen sich die für den StadtUmland-Raum Rostock entwickelten Szenarien von Hagemeier-Klose/Albers/Richter/Deppisch in ihrem Bericht „Szenario-Planung als Instrument einer ,klimawandelangepassten' Stadt- und Regionalplanung - Bausteine der zukünftigen Flächenentwicklung und Szenarienkonstruktion im Stadt-Umland-Raum Rostock“". Allerdings liegt hier der Fokus mehr auf veränderten Bedingungen durch den Klimawandel, denen soziale, ökologische, ökonomische und politische Aspekte hinzugesellt werden, um mögliche Entwicklungen aufzuzeigen. Eine Kerngruppe aus Vertretern der lokalen und regionalen Politik und Verwaltung, unterstützt durch die Wissenschaftler und Wissenschaftlerinnen der HafenCity Universität Hamburg, konzipierte und 
organisierte eine dreistufige Workshop-Reihe, in die ein erweiterter Teilnehmerkreis aus zentralen lokalen Akteuren einbezogen wurde. Auch am Ende dieses Berichts finden sich vier umfangreich beschriebene Szenarien, die durch ansprechende Bilder visualisiert werden.

Wurpts/Kirchgeorg widmen sich im dritten Bericht des vorliegenden Heftes einer ganz anderen Thematik: Clusterförderung im Rahmen der „Gemeinschaftsaufgabe zur Verbesserung der Regionalen Wirtschaftsstruktur". Sie analysieren Konzept und Struktur von 52 in Clustern vereinten Organisationen in Ostdeutschland, die im Rahmen der Gemeinschaftsaufgabe gefördert werden, um im Ergebnis das Förderprogramm und seine Implementierung zu evaluieren. Sie kommen unter anderem zu der kritischen Erkenntnis, dass es an thematischer und geographischer Koordination sowie an einer Evaluierung der Förderung mangele.

Doch lesen Sie selbst! Wir wünschen Ihnen eine angenehme Lektüre. 